\title{
Le repos de sécurité, les 48 heures hebdomadaires et moi
}

\author{
Y. Panis \\ (C) Lavoisier SAS 2017
}

Il y a un peu plus de deux ans, j'avais commis un éditorial dans cette même revue, éditorial qui tentait de démontrer que « de mon temps, c'était bien mieux ». Pour résumer, malgré des journées plus que bien remplies, l'interne de chirurgie digestive de la fin des années 1980 et début des années 1990, apprenait bon an mal an son travail, sans trop se plaindre, avec environ 80 heures de travail hebdomadaire, et était « formaté » pour enchaîner parfois plusieurs journées de suite de travail, notamment le week-end.

Ce mode de fonctionnement avait une logique : celle de la continuité des soins. Ainsi, un interne suivait de $\mathrm{A}$ à $\mathrm{Z}$ les péripéties pré- et postopératoires du patient dont il avait la charge. Non seulement, ce mode de fonctionnement rassurait le patient qui avait « son interne » mais en plus, et surtout, il permettait une formation d'excellence à l'interne, car comme chacun le reconnait, la prise en charge périopératoire est la meilleure école de formation des internes en chirurgie (avec le bloc opératoire évidemment).

L'internat d'hier avait ses morceaux de bravoure comme ces nuits sans sommeil consécutives, ses visites interminables du samedi matin, ces blocs qui n'en finissaient pas et qu'il fallait recommencer chaque jour avant de s'enquiller un samedi et un dimanche de garde à la suite. La geste de l'Internat est remplie de ces moments de fatigue, de passion, et parfois aussi de solitude, voire de détresse. Elle en était l'essence même. C'était, sans exagérer, notre gloire et notre fierté.

Bref, voici en résumé ce que j'avais écrit dans cet éditorial, que presque personne n'avait lu à l'époque, et qui de toutes les façons, à l'évidence, n'était (déjà) plus dans l'air du temps. Mais alors vraiment plus...

Plutôt que de continuer à pleurer sur le passé qui ne reviendra pas (par définition), et parce que le paradigme a totalement changé, il faut bien faire avec et continuer à avancer sagement jusqu'à la retraite...

Et donc aujourd'hui, un interne fait cinq à six gardes par mois, avec donc un repos de sécurité le lendemain, ce qui nous fait cinq à six jours « off » par mois. Il ne fait plus de week-end avec deux jours de garde consécutifs non plus. Ce

\section{Y. Panis $(\bowtie)$}

Hôpital Beaujon, 100 boulevard du Général Leclerc,

92110 Clichy, France

e-mail : yves.panis@bjn.aphp.fr qui est une vaste connerie, car cela gâche finalement sa qualité de vie : au lieu d'un seul week-end « pourri » par mois, il en a aujourd'hui souvent deux, avec une fois un vendredidimanche, et une fois un samedi... Quel progrès... Enfin, pend au nez de l'interne la loi des 48 heures hebdomadaires, garde comprise, qui arrive au grand galop.

Adieu continuité des soins ! Adieu la position centrale de l'interne dans un service de chirurgie ! Demain, il ne sera plus qu'un employé quelconque qu'on appellera, qui pour aller au bloc, qui pour signer une ordonnance, mais à qui on aura du mal à confier le suivi d'un patient, puisqu'il ne sera pas présent chaque jour?

Bientôt, l'interne rentrera dans l'ère formidable des « transmissions », où l'interne « sortant » donnera ses informations à l'interne « entrant ».

Le décret du JO du 3 mars 2015 porte en effet la semaine de l'interne à 48 heures hebdomadaires maximum, garde comprise, sans autorisation de dépasser huit demi-journées hebdomadaires. Mais comme si cela ne suffisait pas a été rajouté : «l'interne bénéficie d'un temps de pause d'une durée minimale (sic) de 15 minutes par demi-journée en stage ». Les pauvres chéris...

Supposons que je sois devenu définitivement un « vieux con » et que donc j'ai totalement tort. Je ne comprends pas le progrès social, je suis un " antimoderne », et des gens comme moi, il y en a toujours eu et ils ont toujours eu tort. OK, j'accepte la critique.

Je rétorque juste, pour essayer de me défendre, la chose suivante : préférez-vous être opéré par quelqu'un qui a 20000 heures de chirurgie ou seulement 10000 heures ? Qui pense raisonnablement que la réduction des heures de formation de l'interne va être associée à une équivalence de formation? Par quel miracle un interne de 25 ans d'aujourd'hui va être formé avec 48 heures hebdomadaires et ce aussi bien qu'un interne d'hier qui arrivait à être pas trop mal formé avec 75 heures hebdomadaires ? Qui peut nous faire croire ça ? Nos CNU ? Nos doyens ? Nos ministres ? Doit-on continuer à se cacher derrière la classique réponse de nos chefs : «c'est à cause de l'Europe »? Bonjour Munich !

La dernière phrase de mon éditorial d'il y a deux ans était : «Ne peut-on pas au moins essayer de négocier avant d'abdiquer? ». Je la laisse car elle reste cruellement d'actualité je trouve... 